\title{
Effect of item length and number of different elements on immediate memory'
}

\author{
JAMES R. WILLIAMS ${ }^{2}$ AND DONALD L. FISH ${ }^{3}$ \\ NEW YORK UNIVERSITY
}

\begin{abstract}
Abstraet
Six Ss were tested for immediate memory on number and symbol items under conditions representing all possible combinations of 2 through 9 item lengths and 2 through 9 different elements. The items (symbol or number) were presented sequentially on a memory drum (the symbols for 3 sec. and the numbers for $1.5 \mathrm{sec}_{0}$ ) and $\mathrm{S}$ recorded what he remembered immediately after the item disappeared from view. The results indicated that short-term retention decreases as the item length increases or as the number of different elements in the item increases.
\end{abstract}

\section{Problem}

The problem concerning the apparent limit to man's short-term memory store capacity has received considerable attention in recent years. Certain authors (Miller, 1956) have felt that these limits are rather fixed, while other authors indicated that changes can occur under certain conditions, such as training, careful selection of stimulus material, etc.

Inherent in the limitation of human short-term memory capacity is the so-called "memory span." In a survey of the literature during the late 30 's, Blankenship (1938) found that three factors seemed to be involved with item difficulty: length of the item, familiarity with the material, and associability. Blankenship also found that grouping had an important effect on memory span (one study showing an increase from 6 to 13.8 units via grouping techniques). A study by Crannel \& Parrish (1957) also found grouping to be important with the retention of digits, but of negative value for the retention of other materials. Crannel and Parrish failed to find a relationship between difficulty in immediate memory and the number of different items from which the $\mathrm{S}$ had to select. This hypothesis was tested, however, by comparing a limiting condition (where the letters or word list was limited to 9 different items) with a non-limited condition, rather than systematically varying the number of different items.

The purpose of this present study was to systematically vary the length of the item as well as the number of different elements within the item and to determine the resultant effect on immediate memory. Symbol as well as number items were investigated in order to determine the difference between familiar and unfamiliar items.

\section{Method}

The experimental apparatus consisted of a memory drum, two symbol item lists, and two number item lists. All of the symbols were easily "verbalized" and were matched for recognizability on the basis of results from a previous study (Williams \& Falzon, 1963).

The following 9 test symbols were utilized: asterisk (X overlaid on a cross), circle, diamond, triangle, square, cross, upside-down $\mathrm{T}$, semi-circle, and a slash. Symbol height was approximately $5 / 16$ in. The numbers used were the digits 2 through 9 and were produced by a standard typewriter. Each list contained 36 items which represented all possible combinations of item lengths (2 through 9) and number of different elements ( 2 through 9). Items and sequence of items were constructed randomly without replacement. Two lists were constructed for each of the item types in order to balance any chance effects.

Due to the nature of the stimulus conditions (the treatment matrix had the form of a half matrix since some conditions were impossible-such as item length of 2 with 9 elements), only item lengths of 6 through 9 and element conditions of 2 through 6 were included in the analysis of variance model to be used for analysis of the experimental results. With the inclusion of the "trials" factor, the resultant model became a 4 by 5 by 2 ANOV. Six Ss (male graduate students) were tested under each of the experimental conditions.

To familiarize the Ss with the stimulus materials, they were given five practice trials before each experimental run (list). The Ss were instructed to write what they had "just seen" in the memory drum and get the symbols or numbers in their proper locations within the item. $\mathrm{S}$ was presented with the items in the list, one at a time. The items on the symbol lists were exposed for $3 \mathrm{sec}$. and the Swas allowed a $12 \mathrm{sec}$. "write" period. The number items were exposed for $1.5 \mathrm{sec}$. and allowed a $6 \mathrm{sec}$. write period. Preliminary investigation indicated that the $3 \mathrm{sec}$. symbol exposure rate was equivalent to the $1.5 \mathrm{sec}$. number exposure rate as far as difficulty was concerned. The S completed the first two lists, was given a 5 min. break, and then completed the last two lists. The lists sequence was presented in a balanced order among Ss to prevent any ordering effects.

\section{Results and Discussion}

The percent of the number of correct digits or symbols, correctly positioned within the item, served as the dependent variable in this study. Table 1 presents the overall means for both numbers and symbols trials on all 36 conditions. By examination of these means, it can be seen that as the length of the item increases, the percent recall decreases; and as the 
number of elements increases, percent recall also decreases.

The results of the analysis of variance for symbol data yielded two significant main effects (item length at .05 and number of elements at .01) and a significant three factor interaction (.05 level). The lack of an interaction between item length and number of elements is easily seen in Fig. 1. The insignificance of the trial factor indicated that neither a practice effect nor a fatigue effect was present in this experiment. Inspection of the data yielded no systematic trends to explain the three factor interaction.

The analysis of variance for the number data also yielded significant $F^{\prime}$ 's (at .05 level) for item length and number of elements, but did not yield a significant three factor interaction. Trials was again not significant. In comparing the two sets of curves in Fig. 1 (symbols vs. numbers), it can be seen that the shapes are very similar except for the greater smoothness and slope exhibited by the symbol curves. Inspection of the curves indicated a marked superiority of the number items for recall even though they were viewed at only half the exposure time. Although not significant, inspection of the number data curves indicated that as item length increased, the number of elements in the item had more effect on recall than was the case for shorter item lengths.

Several other interesting trends were noted during the course of the experiment. The first trend was in respect to "grouping for recall." Ss who grouped numbers by two's seemed to do better than those who grouped by three's or other groupings. The second trend of interest was in relation to the method used by the Ss in recalling symbols. Ss (two in all) who indicated that they did not verbalize the symbols, but rather retained a "mental image" of the material, did better than those who verbalized the symbols and

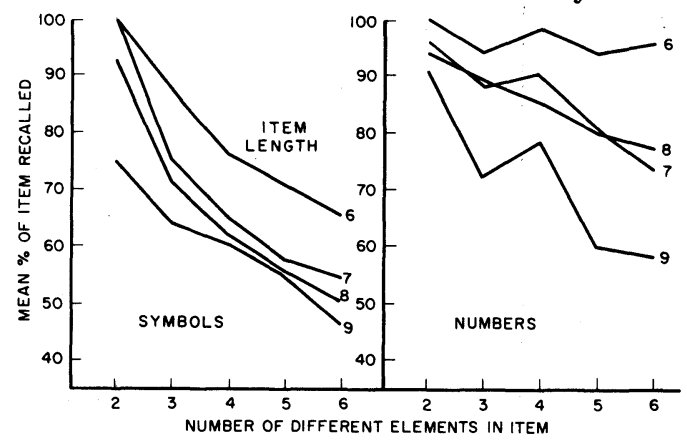

Fig. 1. Effect of item length and number of different elements in the item on immediate memory.
TABLE I

Means for percent correct short term retention

for symbol and number items varying in item length and number of elements.

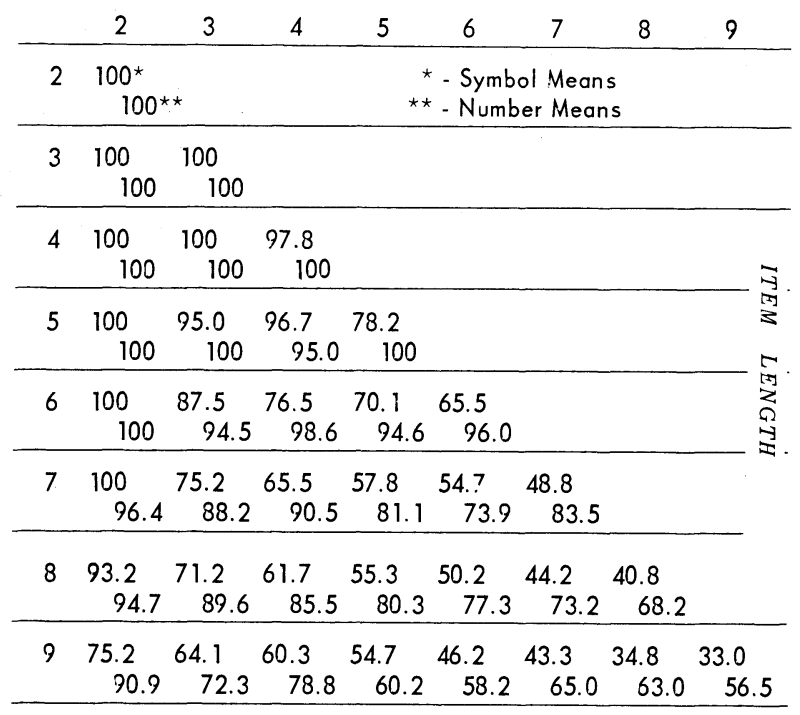

NUMBER OF ELEMENTS IN ITEM

retained the verbalization in memory. This trend is very interesting and may indicate separate stages of information processing in the brain. Implications about new methods of training are also inherent in this trend.

\section{References}

Blankenship, A. B. Memory span: A review of the literature. Psychol. Bull. 1938, 35, 1-25.

Crannel, C. W.. \& Parrish, J. M. A comparison of immediate memory for digits, letters, and words. J.. Psychol., 1957, 44, 319-327.

Lloyd, K. E., Sr. Short-term retention as a function of average storage load J. exp. Psychol., 1961, 62, 632.

Miller, G. A. The magical number seven, plus or minus two; Some limits on our capacity for processing information. Psychol. Rev., 1956, 63, 81-97.

Saul, E. V., \& Osgood, C. E. Perceptual organization of material as factor influencing ease of learning and degree of retention. J. exp. Psychol., 1950, 40, 376-379.

Williams, J. R., \& Falzon, R. P. The relationship of display system variables to symbol recognition and search time.J. Eng. Psychol., $1963,2,97-111$

Winer, B. J. Statistical principles in experimental design New York: McGraw-Hill, 1962.

\section{Notes}

1. This research was performed at New York University, Department of Industrial Engineering \& Operations Research, as part of a course entitled "Research Methods in Human Factors" under the direction of Dr. M. S. Mayzner.

2. Employed by Kollsman Instrument Corporation.

3. Employed by Grumman Aircraft Engineering Corporation. 